\title{
Advanced Driver Assistance System using Convolutional Neural Network
}

\author{
Geetha. A ${ }^{\text {ar, Sharmila. }}$ P $^{\mathrm{b}}$, Sobitha Ahila.Sc, and Raja Senbagam.T ${ }^{\mathrm{d}}$
}

AAssistant Professor,Department of Computer Science \& Engineering, Easwari Engineering College, Chennai
B Assistant Professor, Department of Information Technology, Sri Sairam Institute of Technology, Chennai
C Associate Professor,Department of Computer Science \& Engineering, Easwari Engineering College, Chennai
D Assistant Professor, Department of Computer Science \&Engineering,Government College of Technology, Coimbatore

Article History: Received: 11 January 2021; Accepted: 27 February 2021; Published online: 5 April 2021

\begin{abstract}
Road sign recognition is an essential task in driving process to drive safely and to avoid accidents. Road sign recognition is not a simple task as there are many unfavorable factors such as bad weather, illumination, physical damage etc. The purpose of Road sign is to inform drivers and autonomous vehicles about current state of road and also provide them other important data for navigation. This paper aims to build Convolutional neural network (CNN) model to recognize road signs and to inform the drivers in advance for safe driving. The advantage of using Convolutional neural network (CNN) is its potential to build an internal representation of two-dimensional images. This enables the model to learn scale and position variant structures in the data, which is required when working with images. The proposed system achieves an accuracy of $87 \%$. Keywords: Road sign Recognition, Image Processing, Convolutional neural network, Tensor flow, advanced driver assistance system.
\end{abstract}

\section{Introduction}

Image processing is a computer based technology which performs some operations on images to get improved images or to extract useful data from it. The extracted information is further analyzed using machine learning algorithms to provide desired results. Machine learning algorithms are used to train the models using images and classify them according to the corresponding classes (Aroulanandam, 2020). Road sign recognition has an important role in driver assistance system and intelligent autonomous vehicles. Road signs are fundamental part of road infrastructure. They provide critical information for drivers which help them to adjust their driving behavior to ensure safe driving irrespective of road conditions (Latchoumi, 2020). Due to lack of awareness about signs, a large number of accidents occur. They would not get proper suggestions like how far they could go safely or not informed about road works or school crossings or other important road signs. They easily get distracted in so many ways.

The Road accidents are especially high under special road conditions such as entering in one-way or share curves. One possible solution to avoid this to keep road signs visible on the streets to notify the drivers about the road condition and other road information. However, most of the drivers are not paying attention to these signs while driving due to distraction or carelessness. Predictions on road accidents state that approximately $40 \%$ of the road accidents could be averted by notifying drivers in advance about these road signs. It is possible to prevent accidents by effectively making use of automatic road sign recognition system which provides road information in advance to the drivers. Image processing technology can identify the external environment by means of on-board camera mounted on the vehicle which would reduce the burden on drivers and it is expected to provide an effective approach to recognize road signs using machine learning algorithms thereby improving road safety.

This paper focuses on a system developed using Convolutional neural network (CNN) for sign detection and recognition at a faster and more accurate rate. The system captures images of road signs using camera. The captured images are sent to Raspberry Pi. The processes the captured images using the developed CNN model and detect the class of the respective traffic signs. The recognized road sign is informed to drivers as voice signal in order to make them aware of it. The system architecture is represented in Fig.1. Paper organization is as follows: Section II describes review of literature. Section III describes traffic sign detection method using CNN. Section IV describes experimental results of proposed method. Section V contains conclusion and future works. 


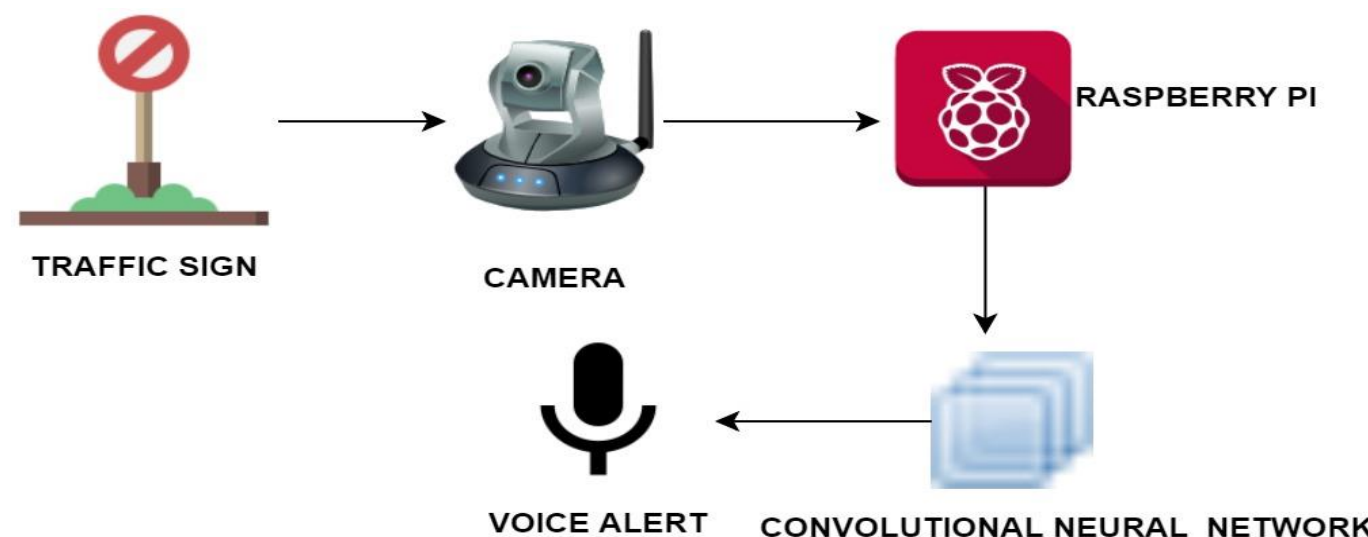

Figure 1. System Architecture

\section{Related Works}

Traffic-sign recognition method are mostly divided into 2 stages: Traffic sign Detection and Recognition. Detection of traffic signs is dependent upon color analysis. In real fact, Traffic Signs have some color specifics such as red border for hazards and warning signs and blue backgrounds for signals. In (Ahmed Madani, 2018; Deepthi, 2020) Ahmed Madani et al, describes that the image boundary colours, based on learning vector quantification (LVQ), were derived using an adaptive image segmentation technique. LVQ is the supervised method of learning that is used to train the prototypes in different color HSV details. After segmentation, a simple and fast matching technique detects the structure of the traffic signals using the exclusive and logical OR operator. Pictogram derived is labeled with Support vector machines (SVM) (Carlos, 2008; Balamurugan, 2020).

The issue of automatic identification of traffic signs from photographs taken while driving is discussed by Carlos Filipe Paulo et al, which can be defined on basis of their color as well as shape and categorized into hazard, data, obligation or prohibition classes. Due to their pictograms, traffic signs may be identified, as every pictogram is special within a given sign class. Suggested method analyzes the exterior contours of the pictogram. The symbols are matched to the database using the curvature display. Various examples of the usefulness of the device are drawn from Portuguese roadways.

In (FatinZaklouta, 2012; Sneha, 2020) they uses descriptors and distance transformations (DT) of differentsized HOG for the classification of traffic signs that do not accommodate image shifts in dynamic environments due to their own design deficiencies that contribute to unsatisfactory accuracy of classification. Results of random forests, $\mathrm{k}-\mathrm{d}$ trees and SVM are analyzed in this work. Random forest reaches maximum precision of up to $97 \%$ by evaluating the performance of all three algorithms. By applying spatial weighting, the $\mathrm{k}-\mathrm{d}$ tree precision is increased by $15 \%$. In addition, the Fisher criteria is tailored to minimize the requirements for memory and computation. This also contributes to improved accuracy (Ezhilarasi, 2020).

HeeSeok Lee et al developed use of convolutionary neural network (CNN) as a new road sign detection system that simultaneously evaluates location, precision and limit of traffic signs. Since traffic signs may be utilized as 3 dimensional road environment landmarks, it is important to estimate the exact limit of traffic signs for intelligent vehicles in navigational systems. A 2-D pose is formulated as the limited estimate of the traffic sign and a single $\mathrm{CNN}$ can resolve the problem of prediction of the form class. By projecting the boundary of a template sign in the input plane with the projected 2-D pose and the shape class of a goal road sign in the input, the real boundary of the target sign can be calculated (Garikapati, 2020). When the boundary estimate problem is formulated as a CNN pose and shape prediction task, this approach is end-to-end training and more robust in the face of occlusion and restricted goals than other boundary estimation approaches that rely on contour estimation or image segmentation. With the CNN-based traffic sign detection network, the proposed method achieves a detection frame rate of more than seven frames per second while delivering very accurate, stable traffic sign detection and border estimation results on a low-power mobile platform (HeeSeok Lee, 2018).

A modern data-driven framework that involves symbol-based signages and text-based signs in frames in video sequence identification of all types of traffic signs provided by Henglian Luo et al.. ROIs from each frame was removed first on gray and normalized RGB channels with maximally stable extreme regions. The proposed multitask CNN, which has a broad variety of data including synthetic signs as well as street views marks, is then used 
to distribute photos of the traffic signs to its respective categories in each image. The post-processing ultimately integrates findings in both systems to settle on identification (HengliangLuo, 2018) .

H.Fleyeh et al. implements own-oriented identification of traffic signs based on invoking PCA method to identify an undefined traffic sign by choosing most successful components of traffic sign images (Fleyeh, 2011). This method was checked on two separate databases of boundaries and speed limit pictograms of traffic signs. Nontraffic signals and obstructed sign artifacts are often used to verify the robustness of this strategy. In order to distinguish the images, Euclidean distance is used. When using occluded signs, a score of 71 was obtained. When the signs were rotated about 10 degrees around the middle, the output was 89 " when the exterior forms of the traffic signs were used.

Jack Greenhalgh et al, explains that candidates should be classified on the basis of the background color of the road signs instead of detecting candidates for road signs by border color, as these backgrounds persist throughout the Maximally stable extreme regions (MSER) phase (Jack Greenhalgh, 2012). Recognition is based upon the use of a HOG, classification of SVM. This machine is reliable at high speeds, worked on a variety of weater settings, runs at a mean speed of 20 images per second and recognizes from an on-line road sign database all forms of ideogram-based (nontext) traffic symbols.

Based on its relative position against the camera and surrounding conditions such as temperature and daylight, much of the road sign changes its form and colour. Thus, Kazumasaet al suggested the object detection using utilized SIFT . Usually, the calculation costs for detecting SIFT and matching with database are expensive. method is applied for traffic sign recognition. At recognition phase, SVM approach is utilized which provides higher accuracy rate . (Kazumasa, 2009)

A vehicle guidance system based on vision must be able to identify and interpret traffic signals. Traffic sign identification systems gather data on road signals to help driver make prompt choices and smoother and faster driving. [9] Kiran C.G et al. clarified the color information to detect and identify traffic signals from image sequences. For traffic sign recognition, color-based segmentation techniques are used. In order to improve segmentation efficiency, the product of increased hue and saturation components was used. Since automated traffic sign detection has been an incredibly difficult process, Mr Hossain and others have suggested a new approach to ADRTS. (SajjadHossain, 2010)

This paper takes moment invariants, color segmentation and NN into account. Color RGB is used in the color segmentation of the traffic sign. A linear SVM was used to boundary features of segmented blobs to achieve improved shape classification efficiency. A non linear vector help multi classifying machine with edge based pixels of interest as property is used for the identification of traffic signals. In various steps the Neural Network Classification is used to identify the various ways and figures within that we make a final decision. The experimental study showed the best efficiency when traffic signals were identified and detected. The complexity of computing time is indeed very limited and refers to the real-time system. Many new machine learning approaches such as Convolutional Neural Network $(\mathrm{CNN})$ with its strong represental capacity and robustness has achieved good results. Qingqing et al, mentioned two defects in identifying existence of traffic signs in real world without eliminating interference data and not fully utilizing hidden data in images which can be solved using CNN with LVQ .CNN is a very efficient algorithm in case of image detection, salient detection and video salient detection(Qingqing, 2018).

In Saturnino Maldonado et al, almost the first stage is based on segmentation stage using RGB to detect strong colors in the surrounding. But the value of hue and saturation in HSI is used to isolate the traffic-signs from video frames. According to the color of pixel, blobs are extracted from input by thresholding for chromatic and non chromatic signs. Using linear SVMs, the classification is based on colors and the shapes representing that colors are classified. After classification, recognition is based on SVMs with Gaussian kernel (Saturnino,2007).

Sunghokimh has the exact effect of a traffic sign region observed on the traffic signs recognition (TSRs) and an enhanced traffic sign sign region extraction method is proposed for TSR. The traditional traffic sign detection (TSD) based on HOG demonstrates poor precision in positioning. Inaccurate sign area directly affects TSR output. Speed limit signals are used with the particular TSR to analyze the impact of a position mistake. The improved TSR performance is validated with the increased color channel-based extraction in TSR training and testing level (Sungho, 2015).

Manisha et al, explains that image segmentation is performed for image enhancement to get clearer data under variable lighting conditions. Mostly images contain dark areas that had significant data. These hidden images are retrieved by changing intensities of images using histogram equalization. Then unwanted areas that do not contain traffic signs are removed and regional coordinates of detected signs are returned to recognition phase where 
ECOC method was utilized to train Multiclass SVM. ECOC reduces classification issue with three or more classes to set binary class(Manisha, 2017).

The new AdaBoost ELM, based multiclass extreme learning machines, is presented by Yan Xu and others. Due to its considerably faster learning speed and much better generalization than, the weighted ELM is selected as basic weak classifier. Then weighted majority of all weighted ELMs constructs an ensemble-strong classifier. Proposed method solves issue in comparison to existing ELM methods of how weighted specimens can be trained directly by ELM in multiclass classifications and reach a high accuracy of $99.12 \%$ in terms of computer complexity, compared to a variety of modern algorithms (Yan, 2016). Yingying Zhu has suggested a new text-based traffic signal-detection framework with the two deep learning components that apply a fully convolutionary network to the traffic sign area candidate segment, with the following: candidate regions of interest (RoI). The suggested approach utilizes the properties of traffic signs in full to boost performance and identification of text. This suggested two-stage detection technique reduces the text recognition search field and eliminates text from traffic signs. It also effectively addresses the multi-scaling issue of the text detection portion. The latest findings from the publicly accessible traffic sign data collection are collected through this method: Traffic Guide Panel data set. Text-based traffic signs were included, including traffic signs in Chinese as well as English. This data collection also indicates that the proposed system is commonly used to identify traffic signals from multiple languages.( YingyingZhu, 2018).

In Yujunzeng et al, suggested using KELM classifier with deep perceptual characteristics, a novel traffic sign recognition method called DP-KELM. (Kiruthika, 2019) KELM classifier is trained with high computational effectiveness and generalization efficiency on basis of learned deep perceptual function. This method has been shown to have higher precision through experiments on German traffic sign recognition benchmark. In fact, a comparable recognition rate with significantly lower calculation costs can be found in comparison to the highest accuracy stochastic gradient lowering method. (Yujun Zeng , 2017)

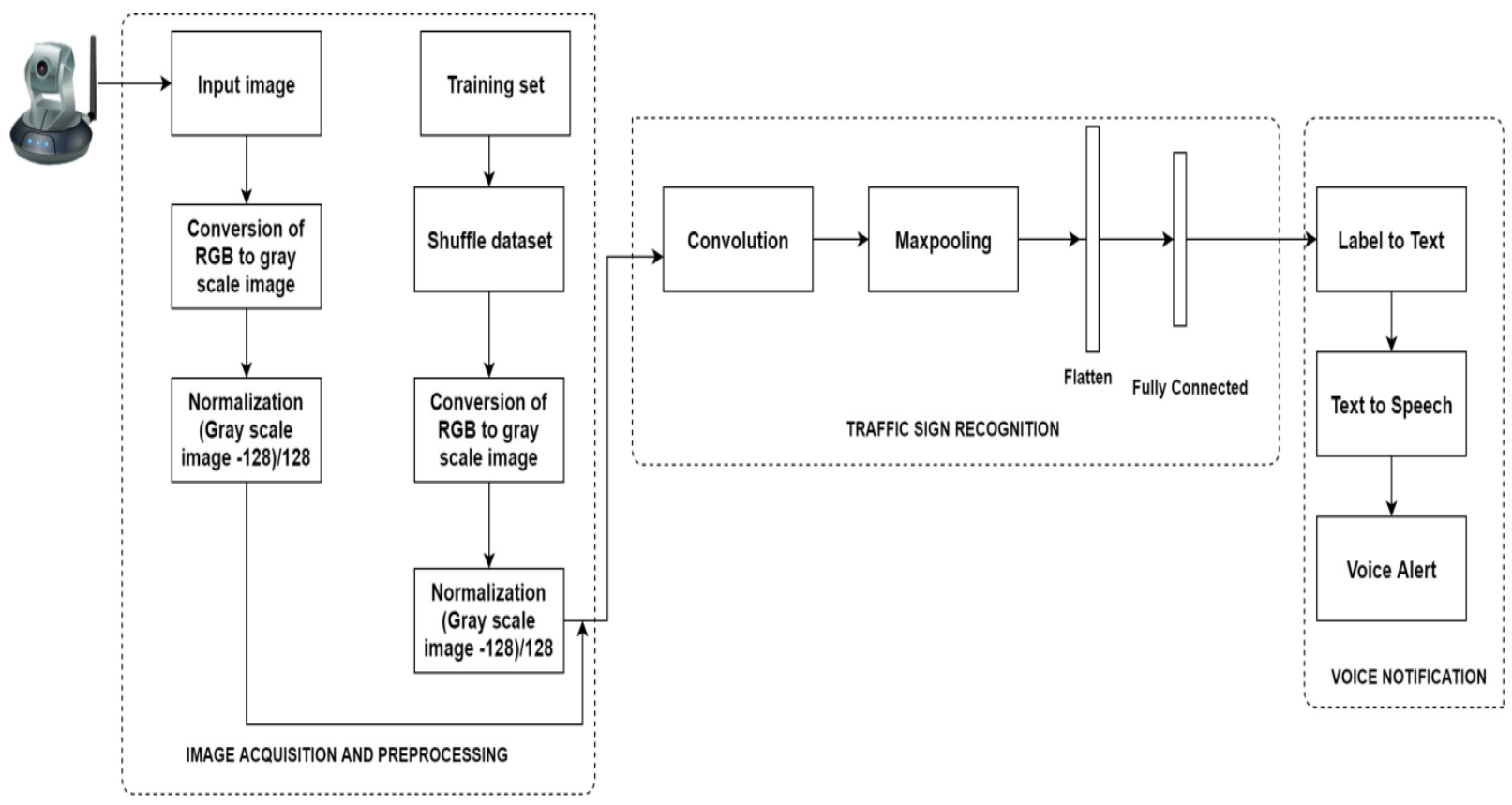

Figure 2. Functional architecture

\section{The Proposed System}

A Robust Traffic sign Recognition accurately recognizes road signs which ensures safety to drivers, passengers and their surroundings. The technological improvement in Traffic sign Recognition system will greatly help to increase the standard of human life. The Proposed System consists of three modules 1)Image Acquisition and Preprocessing 2)Road sign Recognition 3)Road sign Notification which is represented in Fig.2

\subsection{Image Acquisition and Preprocessing}

In this module, the Images of Road signs are captured using camera mounted on the car. The collected data is stored and sent to Convolutional Neural Network(CNN) model with the help of Raspberry pi. The images of road sign needs to be preprocessed before it is fed into CNN model. The Image preprocessing can be done in two steps, 
i) Convert RGB color of the road signs into Grayscale image. In RGB the color reflection illuminates in different light conditions. RGB consists of red, green ,blue which can change it appearance to different color under certain conditions. Conversion of RGB to grayscale changes of illumination can be shown in intensity and the technique is cost efficient.

ii)Normalization is performed on grayscale image. This approach is sometimes called contrast stretching or histogram stretching, changes the pixel intensity values.We use $\mathrm{X}$-wing gray normalization technique as it works well for our model.

\subsection{Road Sign Recognition}

CNN is one of the commonly utilize Deep learning models.CNN has demonstrated high performance on Image classification.Due to its high recognition rate and faster execution, most object recognition are done using CNN. Training of neural network is implemented using keras with backend as tensorflow .The CNN model consists of five layers which is represented in Fig.3,

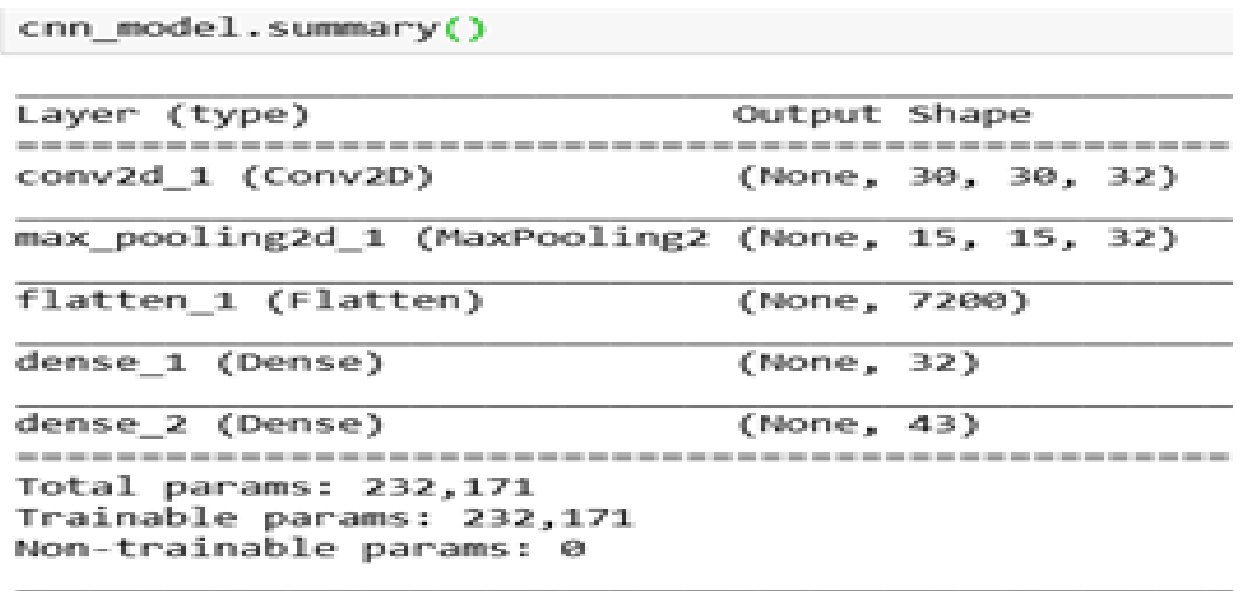

Figure 3. CNN model layer

\subsubsection{Convolutionallayer:}

Convolution is performed to preserve the spatial arrangement on both horizontal and vertical directions. This layer defines weight matrix that acts as a filter in an image extracting particular features from the original images which helps network to make correctprediction.Convolution is always the first layer.The images are entered as matrix with pixel values. This layer chooses a smaller matrix which is called Filter. The role of the filter is to multiply the initial pixel values by its values. All these increases are summarized. At the end of the day there is a figure. Although this filter only has read image in top left corner, 1 unit conducting identical operation pushes further and further to the right. A matrix is obtained, but smaller than an input matrix after the filter is applied over all locations.

\subsubsection{Max Pooling layer:}

The aim of pooling process is to reduce image's spatial scale. It is done independently on the depth of each dimensions and hence depth of image remains unchanged. The pooling layer most widely used is max pooling. The layer of pooling parallels the layer of convolution. It deals with the image width and height as well as executes a retrieval process. This reduces volume of image. This ensures that a complex image is no longer required for further analysis if certain features were already defined during the previous convolution process and are compressed to less detailed images.

\subsubsection{Flattening layer:}

This is the dense layer which converts the output of convolutional layer into 1-D feature vector.This process is known as Flattening.

\subsubsection{Fully Connected layer:}

The output can be produced by applying fully connected layer which generates output equal to number of classes weneed. This model's triggering function is Relu. This function setts zero threshold and looks like:

$f(x)=\max (0, x)$ 
If $x>0$ - volume of array of pixels remains same, and if $x<0$ - it cuts off unnecessary details in channel.

\subsubsection{Output layer:}

The output layer has one output as well as sigmoid activation function. The next step is compiling which uses loss function like sparse categorical cross entropy to compute the error in prediction. Adam, which is ideal for RNN, is optimizer algorithm. Measurements for accuracy indicate the model's efficiency.

\subsection{Road Sign Notification}

In this module, the detected traffic sign data is given as voice alert to the drivers. The Road Sign recognition model recognizes the road signs as labels which is represented in Fig. 4 . These labels are converted to its corresponding classes. The Text-to-Speech API which enables developers to synthesize speech can be used to transform these text into audio.This audio is used as notification to the driver thereby ensuring safety to the drivers

\begin{tabular}{|l|l|}
\hline $\begin{array}{l}\text { Class } \\
\text { Label }\end{array}$ & Description \\
\hline 0 & Speed limit $20 \mathrm{kmph}$ \\
\hline 1 & Speed limit $30 \mathrm{kmph}$ \\
\hline 2 & Speed limit $50 \mathrm{kmph}$ \\
\hline 3 & Speed limit $60 \mathrm{kmph}$ \\
\hline 4 & Speed limit $70 \mathrm{kmph}$ \\
\hline 5 & Speed limit $80 \mathrm{kmph}$ \\
\hline 6 & End of Speed limit $80 \mathrm{kmph}$ \\
\hline 7 & Speed limit $100 \mathrm{kmph}$ \\
\hline 8 & Speed limit $120 \mathrm{kmph}$ \\
\hline
\end{tabular}

\begin{tabular}{|l|l|}
\hline $\begin{array}{l}\text { Class } \\
\text { Label }\end{array}$ & Description \\
\hline 9 & No passing \\
\hline 10 & $\begin{array}{l}\text { No passing for vehicles over } 3.5 \\
\text { metric tons }\end{array}$ \\
\hline 11 & Right of way at next intersection \\
\hline 12 & Priority road \\
\hline 13 & Yield \\
\hline 14 & Stop \\
\hline 15 & No Vehicles \\
\hline 16 & $\begin{array}{l}\text { Vehicles over } 3.5 \text { metric tons } \\
\text { prohibited }\end{array}$ \\
\hline 17 & No entry \\
\hline
\end{tabular}

\begin{tabular}{|l|l|l|l|}
\hline $\begin{array}{l}\text { Class } \\
\text { Label }\end{array}$ & Description & $\begin{array}{l}\text { Class } \\
\text { Label }\end{array}$ & Description \\
\hline 18 & General caution & 27 & Pedestrians \\
\hline 19 & Dangerous curve to the left & 28 & Children crossing \\
\hline 20 & Dangerous curve to the right & 29 & Bicycles crossing \\
\hline 21 & Double curve & 30 & Beware of ice/snow \\
\hline 22 & Bumpy road & 31 & Wild animals crossing \\
\hline 23 & Slippery road & 32 & End of all speed and passing limits \\
\hline 24 & Road narrows on the right & 33 & Turn right ahead \\
\hline 25 & Road work & 34 & Turn left ahead \\
\hline 26 & Traffic signals & 35 & Ahead only \\
\hline
\end{tabular}




\begin{tabular}{|l|l|}
\hline $\begin{array}{l}\text { Class } \\
\text { Label }\end{array}$ & Description \\
\hline 36 & Go straight or right \\
\hline 37 & Go straight or left \\
\hline 38 & Keep right \\
\hline 39 & Keep left \\
\hline 40 & Roundabout mandatory \\
\hline 41 & End of no passing \\
\hline 42 & $\begin{array}{l}\text { End of no passing by vehicles over } 3.5 \\
\text { metric tons }\end{array}$ \\
\hline
\end{tabular}

Figure 4. Traffic Sign Classes

\section{Experimental Results and Discussion:}

This section will calculate proposed traffic sign recognition system on a German Traffic Sign Recognition dataset(GTSRD). Firstly, a brief description about data set is given. Then parameters required for every module in this system are calculated. Finally, the corresponding result for this model is reported. Performance of proposed system is calculated with GTSRD datasets which consists of 34, 799 images for training and 12,630 images for testing. There are 43 classes of signs in this dataset as shown in Fig.4.This dataset give access to static images of symbol-based Traffic signs which are resized to 32 X 32 and are shuffled to train our CNN model. The images in this datasets are represented in 3 channels(RGB).

Since the images in this datasets are differed in their brightness, we use single channel of this image i.e. Grayscale conversion and then normalize these images for better representation.
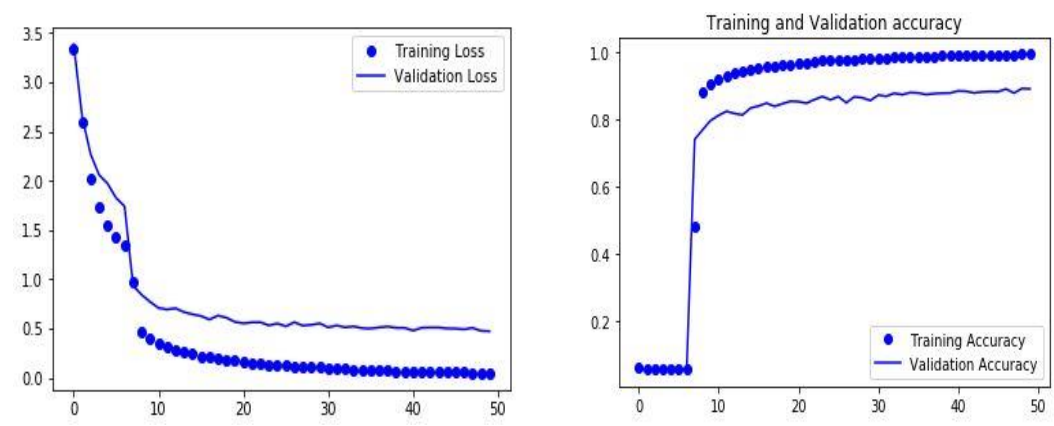

Figure. 5 Training and Validation Accuracy

Our task is to recognize 43 classes of Traffic signs which is done by our CNN model.The Traffic sign recognition system is written in python with Keras which uses tensorflow as its backend.This model is trained with 50 epochs and its batch size is 500.The training time takes around 20-30 mins.Our model achieved an accuracy rate of $87 \%$. The graph is plotted to represent the training and validation accuracy which is shown in Fig.5.

A Confusion matrix is created to calculate performance of proposed technique.This table layout also known as error matrix allows the visualization of the performance of the algorithm. For training phase,our model is run on Pc with keras using tensorflow as background.The model is trained with the minimum learning rate of 0.001 . The system consists of 8GB RAM and Intel core i5 processor which provides very good support to Tensorflow. Although our system provides high performance, there is also mispredictionin some traffic sign images which is shown in Fig.6. 

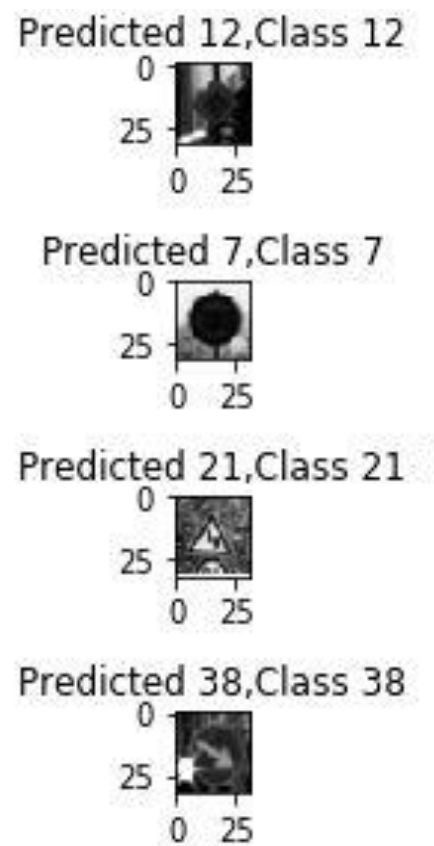
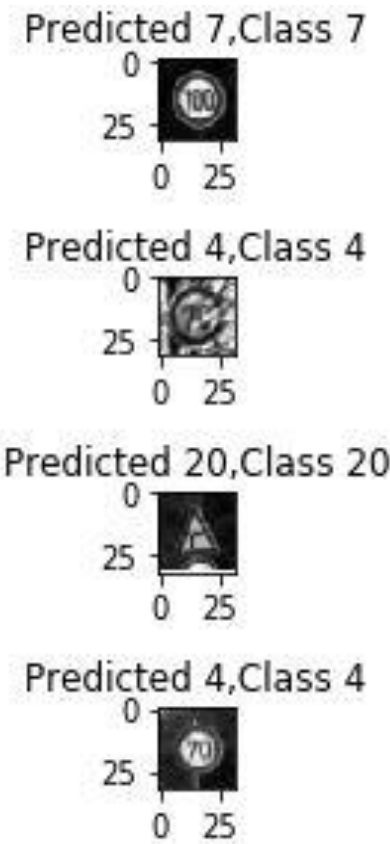
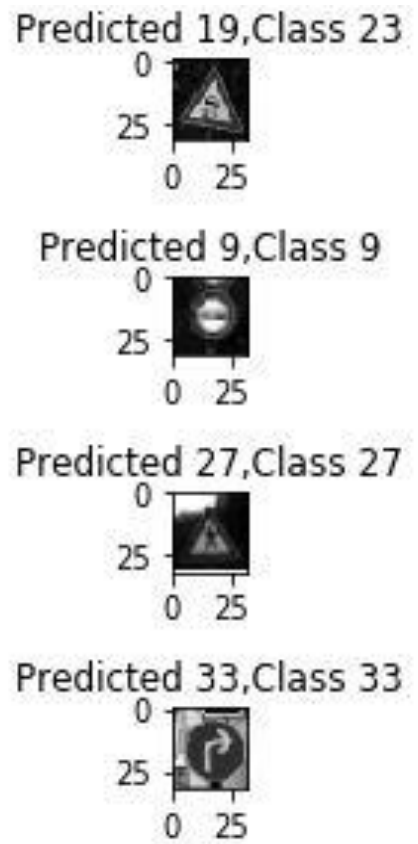

Figure.6 Images of Correctly classified and misclassified classes

Our proposed system provides good accuracy rate of $87 \%$ with simple CNN architecture. To improve the accuracy, techniques like augmentation and flipping can be used. Further, regularization techniques like dropout can be used to minimize the loss rate. Since most of the existing system suffers in memory and processing limitations, our model can be deployed in GCP platform.

\section{Conclusion}

Although many existing system achieved high accuracy rate, the total number of traffic sign classes are very limited and thus they were less likely to suffer from mismatches among similar signs. However, our system uses 43 classes of traffic signs.Most classifiers are trained using hand-labeled real images for example which is repetitive, time-consuming and error-prone process. This method avoids collecting and manually labeling training data since training classification module requires only synthetic graphical representation of traffic signs which is obtained from online dataset.CNN achieved an accuracy of $87 \%$.

\section{Future Works}

The future work focuses on improving the accuracy rate by increasing layers of convolution and pooling. Further, the text based signs along with symbol based signs on the street views can be also be detected. It is possible to extend the suggested methodology to various datasets and observe the effects. Check for widespread possibilities to make it possible to identify any TS from any other country, and not just Germany, without modifying trained dataset. A further study path is to create a road sign recognition device, which captures, identifies and recognizes traffic signals in real time, which provides the driver with an appropriate time frame to respond. In addition, a video is taken by a camera mounted on a car. In real-time implementations, the main problem is the time wasted on traffic sign identification. The collection of suitable strategies for real-time applications and by optimizing the code should minimize this to a minimum. In this thesis, the approaches presented can be changed to satisfy the real-time needs. The benefit of monitoring the sign is that it can be followed in subsequent frames where the sign is occluded or vanished in other frames. If a device of this type is combined with a GPS, it can be used to warn driver about real speed limits on a given route. Driver will be warned when speed limit is breached or the driver does not halt at a STOP sign, by matching the signed limit with the GPS speed reading.

\section{References}

1. Ahmed Madani, RubiyahYusof (2018) Traffic sign recognition based on color, shape, and pictogram classification using support vector machines" , Neural computing and applications, Springer Link, 30 , (9): 2807-2817.

2. Aroulanandam VV, Latchoumi TP, Balamurugan K, Yookesh TL. (2020) Improving the Energy Efficiency in Mobile Ad-Hoc Network Using Learning-Based Routing, Revue d'Intelligence Artificielle, 
Vol 34(3), pp. 337-343. DOI: https://doi.org/10.18280/ria.340312

3. Balamurugan K. Metrological changes in surface profile, chip, and temperature on end milling of M2HSS die steel. International Journal of Machining and Machinability of Materials, 22(6):443-453.

4. Carlos Filipe Paulo, Paulo LobatoCorreia (2008) Traffic Sign Recognition Based on Pictogram Contours",IEEE Ninth International Workshop on Image Analysis for Multimedia Interactive Services.

5. Deepthi T, Balamurugan K, Balamurugan P. (2020) Parametric Studies of Abrasive Waterjet Machining parameters on Al/LaPO4 using Response Surface Method. InIOP Conference Series: Materials Science and Engineering 2020 Dec 1 (Vol. 988, No. 1, p. 012018). IOP Publishing.

6. Ezhilarasi TP, Dilip G, Latchoumi TP, Balamurugan K. (2020) UIP-A Smart Web Application to Manage Network Environments. InProceedings of the Third International Conference on Computational Intelligence and Informatics, pp. 97-108, Springer, Singapore.

7. Fatin Zaklouta, Bogdan Stanciulescu (2012) Real-Time Traffic-Sign Recognition using Tree Classifiers", IEEE Intelligent Transportation Systems Society, 13 (4) :1507 - 1514.

8. Garikapati P, Balamurugan K, Latchoumi TP, Malkapuram R. (2020) A Cluster-Profile Comparative Study on Machining AlSi 7/63\% of SiC Hybrid Composite Using Agglomerative Hierarchical Clustering and K-Means. Silicon. Jun 3:1-12.

9. HeeSeok Lee , Kang ,Kim (2018) Simultaneous Traffic Sign Detection and Boundary Estimation Using Convolutional Neural Network”, IEEE Transactions on Intelligent Transportation Systems, 19 (5) : 16521663.

10. HengliangLuo, Yi Yang, Bei Tong, Fuchao Wu, and Bin Fan (2018) Traffic Sign Recognition Using a Multi-Task Convolutional Neural Network” IEEE Transactions on Intelligent Transportation Systems , 19 (4):1100-1111.

11. Fleyeh, H., Davami, E. (2011) Eigen-based traffic sign recognition”, IET Intelligent Transport Systems, 5(3): $190-196$

12. Jack Greenhalgh, Majid Mirmehdi (2012) Real-Time Detection and Recognition of Road Traffic signs", IEEE transactions on Intelligent Transportation Systems, 13(4):1498-1506. KazumasaOhgushi „Nozomu Hamada (2009) Traffic sign Recognition by Bags of Features”, TENCON 2009 - 2009 IEEE Region 10 Conference.

13. Kiran C.G. , Lekhesh V. Prabhu, Abdu Rahiman V ,; Rajeev K., (2009) Traffic Sign Detection and Pattern Recognition Using Support Vector Machine”,IEEE2009 Seventh International Conference on Advances in Pattern Recognition.

14. Latchoumi TP, Reddy MS, Balamurugan K. (2020) Applied Machine Learning Predictive Analytics to SQL Injection Attack Detection and Prevention. European Journal of Molecular \& Clinical Medicine.;7(2); pp 3543-3553.

15. Qingqing Zheng, Xiaolan Xie (2018) Traffic Sign Recognition Based on Learning Vector Quantization and Convolution Neural Network”, Association for Computing Machinery (ACM) ,:178-183.

16. Sajjad Hossain ,M.,. Mahmudul Hasan , M , Ameer Ali , M,. Humayun Kabir, Md., A B M Shawkat Ali, (2010) Automatic detection and recognition of traffic signs", 2010 IEEE Conference on Robotics, Automation and Mechatronics, IEEE, June 2010.

17. Saturnino Maldonado-Bascón, Sergio Lafuente-Arroyo, Pedro Gil-Jiménez, Hilario Gómez-Moreno and Francisco López-Ferreras (2007) Road-Sign Detection and Recognition Based on Support Vector Machines", IEEE Transactions on Intelligent Transport System, 8 ( 2) :264-278.

18. Sneha P, Balamurugan K, Kalusuraman G. (2020) Effects of Fused Deposition Model parameters on PLA-Bz composite filament. InIOP Conference Series: Materials Science and Engineering 2020 Dec 1 (Vol. 988, No. 1, p. 012028). IOP Publishing.

19. Sungho Kimh and Soon Kwon (2015) Improvement of traffic sign recognition by accurate ROI refinement", Department of Electronic Engineering,2015 15th International Conference on Control, Automation and Systems (ICCAS 2015), October,2015.

20. UKDN Manisha, Liyanage, S.R., (2017) An online traffic sign recognition system for intelligent driver assistance”, 2017 Seventeenth International Conference on Advances in ICT for Emerging Regions (ICTer) , IEEE, September 2017.

21. Yan Xu ,Quanwei Wang, Zhenyu Wei , Shuo Ma (2016) Traffic sign recognition based on weighted ELM and AdaBoost" IEt Electronics Letters, 56 (24):1988-1990.

22. Yingying Zhu ,Minghui Liao, Mingkun Yang, and Wenyu Liu, Senior Member (2018) Cascaded Segmentation-Detection Networks for Text-Based Traffic Sign Detection”,IEEE Transactions on Intelligent Transportations Systems,. 19 (1).

23. Kiruthika, Usha, S. Kanagasuba Raja, R. Jaichandran, and C. Priyadharshini (2019) Detection and Classification of Paddy Crop Disease using Deep Learning Techniques.

24. Yujun Zeng, Xin Xu, Dayong Shen, Yuqiang Fang, Zhipeng Xiao (2017) Traffic Sign Recognition Using Kernel Extreme Learning Machines With Deep Perceptual Features, IEEE Transactions on transportation systems" 18 , (6) 1647-1653. 
